\title{
La investigación de la industria farmacéutica: ¿̨condicionada por los intereses del mercado?
}

\section{Ricardo Páez Moreno ${ }^{1}$}

Resumen: La investigación patrocinada por la industria farmacéutica obedece a intereses científicos de la más alta calidad, pero a la vez a una serie de intereses económicos, puesto que al ser financiada por grandes corporativos transnacionales debe sujetarse a las leyes impuestas por el mercado. En este artículo se cuestiona en qué medida esta dinámica condiciona la integridad científica y la búsqueda de tratamientos para las grandes necesidades en salud. A través de la revisión de la literatura se constata cómo la innovación científica ha quedado disminuida y las prioridades de investigación condicionadas por el mercado.

Palabras clave: industria farmacéutica, ensayos clínicos, integridad científica, intereses económicos

\section{Pharmacy industry research: conditioned by market interests?}

\begin{abstract}
Research sponsored by pharmacy industry obeys to scientific interests of highest quality, but at the same time to several economic interests, since being funded by transnational corporations must be subjected to rules imposed by market. This article questions the measure in which this dynamic conditions scientific integrity and the search for treatments for great health needs. Through literature review, it is shown how scientific innovation has diminished and research priorities are conditioned by market.
\end{abstract}

Key words: pharmacy industry, clinical trials, scientific integrity, economic interests

\section{A pesquisa da indústria farmacêutica: condicionada pelos interesses do mercado?}

Resumo: A pesquisa patrocinada pela indústria farmacêutica obedece a interesses científicos da mais alta qualidade, porém em vista de uma série de interesses econômicos, posto que ao ser financiada por grandes corporaçóes transnacionais deve sujeitar-se às leis impostas pelo mercado. Neste artigo se questiona en que medida esta dinâmica condiciona a integridade científica e a busca de tratamentos para as grandes necessidades de saúde. Através da revisão da literatura se constata como a inovação científica tem se diminuído e as prioridades de pesquisa condicionadas pelo mercado.

Palavras-chave: indústria farmacêutica, ensaios clínicos, integridade científica, interesses econômicos

\footnotetext{
${ }^{1}$ Licenciado en Medicina, Magíster en Bioética, Doctor en Ciencias. Programa de Maestría y Doctorado en Humanidades en Ciencias de la Salud, Universidad Nacional Autónoma de México, México

Correspondencia: ricardomsps@yahoo.com
} 


\section{Introducción}

La investigación de nuevos medicamentos se ha caracterizado por sus altos estándares de calidad y por la neutralidad científica que ha conseguido, particularmente a través de los ensayos clínicos controlados. La industria farmacéutica (IF) se ha preciado por estar a la vanguardia en investigación científica y la eficacia y seguridad de sus productos se ha garantizado por los estrictos controles puestos por organismos tales como la Food and Drug Administration (FDA) y la European Patent Office (EPO)(1).

Sin embargo, la IF está sometida a las leyes del mercado, las cuales, como ha demostrado la historia reciente, forman parte de un "capitalismo desbocado", por los excesos cometidos en diversos frentes y cuya gran crisis es evidente. La industria se ha caracterizado por ser una de las empresas más rentables del sistema de mercado. En la industria, investigación y desarrollo $(\mathrm{I}+\mathrm{D})$ son piezas claves, puesto que de ellos depende la generación de conocimiento para nuevas terapias o para mejorar las ya existentes. La IF justifica su proceder mercantilista por los altos costos erogados en I+D y debido a la competencia entre diversas firmas de la cual no puede salirse. Sin embargo, el riesgo es quedar a merced de los dictados propios del mercado, los cuales pueden interferir con los fines de la investigación científica, uno de los cuales es crear conocimiento para remediar las grandes necesidades en $\operatorname{salud}(2-4)$.

Ante la estrecha relación existente entre IF y mercado, en este artículo se pregunta: ¿en qué medida el interés económico de la investigación patrocinada por la IF empaña la neutralidad científica y la búsqueda de soluciones para las grandes necesidades en salud? Para ello se describirá cómo funciona la IF dentro de la lógica del mercado. En seguida, se abordará el caso de los ensayos clínicos controlados, preguntándose al servicio de quién están. Se tocará el tema de los ensayos clínicos multinacionales multicéntricos, dada su multiplicación en países emergentes como México, que tienen grandes necesidades en salud. Por último, se mencionarán algunos de los sesgos que pueden presentarse con la farmacogenética, que es la investigación del futuro.

\section{La industria farmacéutica como empresa mer- cantil}

La IF representa a las distintas compañías farmacéuticas que tienen entre sus objetivos la $\mathrm{I}+\mathrm{D}$ de nuevos medicamentos. Las medicinas modernas pueden manufacturarse sólo a través de $\mathrm{I}+\mathrm{D}$ complejos y en múltiples etapas. La IF une bajo el mismo techo todas las funciones necesarias para el descubrimiento, desarrollo y manufactura de un medicamento.

Sin embargo, estas tareas farmacéuticas son realizadas por empresas lucrativas, públicamente negociadas y obligadas con sus accionistas. Esto significa que deben tener una ganancia en vistas a compensar el capital invertido en ellas. De esta manera, el descubrimiento y el desarrollo de nuevos medicamentos por la IF están obligados a servir no solo a intereses científicos o médicos, sino también a la eficiencia económica. En otras palabras, la industria debe descubrir y desarrollar medicamentos que "valgan la pena" $(1,4)$.

Según J. Drews, quien fue líder en investigación de Hoffmann-La Roche, la IF tiene que vérselas con dos tipos de criterios que deben satisfacer los medicamentos actuales: científicos y económicos. A la primera categoría pertenecen los criterios de efectividad y seguridad de los medicamentos; a la segunda, aspectos económicos tales como que el medicamento en cuestión sea efectivo contra una enfermedad con una alta incidencia en la población, tales como las enfermedades cardíacas y circulatorias, el asma bronquial, la osteoporosis, varios cánceres, artritis reumatoide primaria y otras enfermedades autoinmunes. Entonces, el tamaño de la población juega un rol decisivo en la decisión a favor o en contra del desarrollo de un medicamento. Sin embargo, esto ha traído conflictos con pacientes o grupos representantes de portadores de enfermedades poco comunes.

Respecto al proceso de $\mathrm{I}+\mathrm{D}$, la creación de un nuevo producto corresponde a una primera etapa, mientras que su desarrollo como fármaco utilizable médicamente tiene que ver con un segundo momento. El éxito definitivo de una nueva molécula depende no solo de su originalidad y propiedades atractivas, sino también de si es puesta rápidamente en el mercado. No basta con inno- 
var, sino que hay que preservar la novedad a través del desarrollo. En el caso de compuestos exitosos, ahorrar un año representa varios cientos de millones de dólares de ganancias. En vistas a acelerar lo más posible el proceso de desarrollo, en lugar de funcionar secuencialmente, una etapa después de otra, se busca trabajar en paralelo para ahorrar el mayor tiempo posible(1).

Para la gente de negocios, una nueva medicina es "buena" solo si está dirigida hacia una indicación económicamente redituable. No se comprometen con estrategia alguna de investigación con resultados inciertos que, aunque no sea muy costosa, consuma mucho tiempo. En la IF se da entonces una tensión entre la innovación de los científicos y la resistencia conservadora de los hombres de negocios que buscan optimizar ganancias.

La IF justifica sus altos precios porque requiere de grandes ventas y ganancias para justificar los altos costos en $\mathrm{I}_{+} \mathrm{D}^{2}$. La reducción de costos junto con los requerimientos del capital pueden ser reconciliados solo cuando se venden grandes cantidades de medicinas. Esto es posible solo en el caso de enfermedades comunes, con bajo costo de manufactura.

Ante las dificultades de crecimiento significativo que ha experimentado la IF, ha sido necesario incrementar la productividad investigativa a través de medidas externas, como las fusiones y adquisiciones de otras empresas que le den fuerza a los productos puestos en el mercado frente a la competencia, guiando a los médicos y pacientes sobre cómo mejor usar el medicamento, y a través de la combinación de varios blancos (derivados de la investigación genómica) con la química combinada y una elevada investigación, lo que proveerá un gran número de "hits". Sin embargo, también son necesarias las medidas internas, tales como aumentar la calidad y cantidad de innovación de productos disponibles cada año para el desarrollo, más unidades de investigación que

\footnotetext{
${ }^{2} \mathrm{La} \mathrm{I}+\mathrm{D}$ es muy costosa y los gastos han crecido más rápido que otros. La razón, además de la inflación en los costos de investigación en Estados Unidos, está en la necesidad del continuo mejoramiento en los métodos investigativos, requiriendo equipo cada vez más nuevo, eficiente y costoso. El costo de encontrar y desarrollar un nuevo medicamento se ha elevado desde 24,4 millones de dólares (1956-1966) hasta 350-500 millones (1996). Sin embargo, como la IF debe ajustar sus aumentos a no mayores al 6\% en Estados Unidos, debe entonces aumentar su producción de nuevas medicinas.
}

dividan la tarea investigativa de una gran firma en pequeñas unidades independientes, colaboración con pequeñas firmas de biotecnología, entre otras. Referente a esta última, Drews afirma que "la industria de la biotecnología bien puede desarrollarse en los próximos diez a quince años dentro de una amplia industria de la investigación que provea sustancias para el desarrollo a una industria farmacéutica que, mientras tanto, se ha llegado a especializar en el desarrollo y la distribución" (1:214).

Más aún, la IF ha sufrido una reciente disminución en la innovación de productos que tiene que ver con su relación con el mercado, la cual se caracteriza actualmente por "la planeación y la mercadotecnia precomercial" (5). Esta estrategia tiene la finalidad de acortar el periodo en que una nueva molécula es aprobada y así aumentar el tiempo de duración de la patente y originar mayores ganancias. Consiste en un cambio en el modelo tradicional investigación, desarrollo y comercialización, por uno nuevo consistente en integrar la investigación de mercado con la investigación, desarrollo y comercialización. Más aún, la integración de la mercadotecnia e $\mathrm{I}+\mathrm{D}$ está dirigida a la creación de valor interno, que tiene un espejo externo llamado demostración del valor. Se trata de integrar diversos equipos de investigación, pero también de mercadotecnia, que demuestren la eficacia y seguridad del producto por nacer y hagan que este sea esperado por todo un público (médicos, pacientes, farmaceutas, etc.) expectante. El esquema actual sería: creación de valor/ demostración, I+D, y control por el mercado(5).

Como puede verse, detrás del interés científico de la investigación proveniente de la IF está el interés mercantil: investigar en enfermedades que afecten a muchos que son las que "valen la pena" (no así las que representen mayor carga de enfermedad), optimizar el tiempo para ahorrar millones de dólares, lograr comercializar un medicamento antes que la competencia, fusionarse para salir adelante, etc. El mayor gasto de la IF no está en I+D, sino en comercialización y mercadotecnia de sus productos. Si bien todas estas pueden ser estrategias válidas de mercado, es claro que la voracidad mercantil propia de un capitalismo desbocado, como al que se está asistiendo, desluce la misión científica de la investigación farmacéutica. Como 
ya apuntaba Drews, lo económico determina las prioridades de la IF: a la hora de fijar prioridades en investigación en salud, estas quedan dictadas por la lógica de la empresa capitalista, cuyo primer objetivo es el lucro y no la satisfacción de las necesidades de la sociedad ${ }^{3}$.

\section{Los ensayos clínicos como estrategia mercantil de la IF}

A raíz de los accidentes como consecuencia de los nuevos medicamentos o vacunas (ciertas epidemias de tétanos causadas probablemente por una vacuna antivariólica en Estados Unidos, en 1901; la comercialización de sulfonamidas en forma de jarabe en excipiente de glicol que causó la muerte de más de 100 personas, también en Estados Unidos en 1937), el Congreso autorizó a la Food and Drugs Administration (FDA) a controlar la toxicidad de los nuevos medicamentos. Esta asumió su papel como controladora del riesgo/beneficio de los nuevos medicamentos que se aprobaban en la medida en que fueran útiles para los pacientes, que no tuvieran tantos riesgos como para sobrepasar los beneficios y que no fueran tóxicos.

Sin embargo, poco a poco el control del cociente riesgo/beneficio fue delegado por los poderes públicos a la IF, debido a las enormes presiones para mantener a la investigación en el marco de la libre empresa de parte de los industriales norteamericanos. Se llegó al grado de que los poderes gubernamentales se limitaban a revisar los resultados, pero dejando total autonomía a la IF. Debido el enorme éxito de las sulfonamidas, los ensayos clínicos lograron regular el desenfreno en la investigación y adquirieron carta de ciudadanía en la investigación farmacéutica(3). Es más, los ensayos clínicos pronto pasaron de ser una herramienta de regulación a ser el corazón del proceso de invención en sí.

Por otra parte, el año 1980 fue clave para un cambio más en la manera de conducir los ensayos clínicos: ahora sería la IF la gran patrocinadora

\footnotetext{
3 Se ha demostrado que la IF antes de investigar en determinado producto, se pregunta por su valor actual ajustado por riesgo, net present value risk-adjusted, NPVr o sea, el monto de invertir más la renta presunta, menos cualquier pérdida de ingresos. Si para un anti infeccioso su NPVr es de 100, para un medicamento oncológico es de 300, para uno neurológico de 700 y para uno del sistema musculoesquelético de 1.150, está claro en cuál de ellos se invertirá. Queda así una población importante de medicamentos huérfanos.
}

y conductora de la investigación. Antes de esta fecha los investigadores eran bastante independientes de las compañías que patrocinaban su trabajo: la IF daba una concesión a algún centro de investigación médica y se retiraba y aguardaba a que los investigadores obtuvieran los resultados. La IF esperaba que su producto fuera exitoso, pero no lo tenía asegurado; no intentaba decirle a los investigadores cómo tenían que desarrollar el ensayo clínico.

La razón del cambio estuvo en el gran crecimiento de las ganancias, poder e influencia de la IF a partir de 1980. Conforme la IF se volvió más poderosa y orientada por las ganancias, su capacidad de esperar resultados de los centros académicos fue menor. Por una parte, los ensayos clínicos disminuían parte de la vida de la patente de los medicamentos ${ }^{4} y$, por otra, solo esperar resultaba muy incierto. Los hallazgos de la investigación podrían resultar contra los intereses de la IF. Por esta razón, la IF contrató a las contract research organizations (CRO), quienes se contratan con médicos privados para recolectar datos de sus pacientes en sus consultorios, de acuerdo con las instrucciones de la compañía patrocinadora. Sin embargo, los médicos no son investigadores, sino que simplemente hacen lo que se les pide. Las CRO le responden sólo a la IF y esta tiene el control sobre estos ensayos. En EU, las relaciones entre la academia y la IF cambiaron a partir de 1980, no solo por la nueva ley Bayh Dole sino también por la necesidad de competir con las CRO, acomodándose a las necesidades de la IF y abriéndose a los grandes financiamientos de ésta.

Como resultado, las compañías farmacéuticas ahora diseñan los ensayos clínicos que deberán poner en práctica los investigadores, quienes son reducidos a ejecutores, ya sea en los centros académicos o en el consultorio privado. La IF analiza e interpreta los resultados, y decide si son o no

\footnotetext{
${ }^{4}$ La patente se otorga por 20 ańos a partir del momento en que se registra una molécula a investigar ante la FDA. Entre más tarde un ensayo clínico, menos durará la comercialización del producto bajo la patente.

${ }^{5}$ Se trata de una ley que facultó a las universidades y pequeños negocios para patentar descubrimientos provenientes de la investigación patrocinada por los Institutos Nacionales de Salud (NIH) -que hasta entonces estaba abierta al domino público para el uso de cualquier compañía que quisiera-, y consesionar licencias exclusivas a las compañías farmacéuticas. En adelante, las universidades, donde se realiza la mayoría de la investigación patrocinada por los $\mathrm{NIH}$, podían patentar sus descubrimientos y cobrar derechos.
} 
publicables(2). El interés financiero se ha asociado a restricciones en la publicación de los datos, particularmente cuando estos son negativos, o a la promoción de beneficios no demostrados, actuando en contra de la generación del conocimiento y obstaculizando la cooperación entre industria y $\operatorname{academia}(6,7)^{6}$.

Ahora bien, a pesar de que se sabe del aumento del impacto potencial de conflictos de interés financieros en la investigación biomédica, casi no se ha hecho ninguna síntesis comprehensiva del cuerpo de la evidencia referente a estos. Bekelman et al.(6) revisaron ampliamente estudios clínicos en la literatura, entre 1980 y 2002, que tuvieran datos originales, cuantitativos, sobre las relaciones financieras entre industria, investigadores científicos e instituciones académicas. Encontraron una asociación estadísticamente significativa entre patrocinio y conclusiones favorables a la IF. Por la vasta revisión de la literatura, concluyeron que la relación entre industria, centros de investigación e investigadores es dominante.

"La evidencia fuerte y consistente muestra que la investigación financiada por la industria tiende a producir conclusiones a favor de esta. Combinando datos de los artículos que examinan los 1.140 estudios, encontramos que los estudios financiados por la industria fueron significativamente más propensos a alcanzar conclusiones favorables al patrocinador que en el caso de estudios no patrocinados por la industria(6:463)".

En cuanto a la calidad de los estudios, la industria los realiza en alto grado a través de metodologías como la aleatorización y el cegamiento. Sin embargo, olvida importantes herramientas que pueden ser usadas en su contra, como la relevancia de la pregunta de investigación. En efecto, en

\footnotetext{
${ }^{6}$ La IF ha mantenido un estricto control sobre los datos recogidos, lo cual ha sido utilizado a su favor. Además, algunos ensayos clínicos tienen sus propios comités de publicación, controlados por investigadores internos o externos, que escriben los resultados a publicarse. Otras veces, las redes comerciales de medicamentos escriben los reportes para publicación, designando a los autores según hayan participado en el diseño del estudio, hayan contribuido a reclutar más pacientes o tengan buena fama en el campo. Los resultados son publicados si favorecen a la IF, y ofrecen problemas cuando van en sentido contrario. Estudios más actuales en la misma línea han sobreabundado en la literatura, mostrando la fuerte influencia de la IF sobre la investigación. Bodenheimer ha demostrado varios casos de sesgo informativo en los resultados sobre investigación en medicamentos patrocinados por la IF(8-11).
}

orden a obtener financiamiento, los investigadores han privilegiado estudiar nuevos fármacos $\mathrm{e}$ instrumentos diagnósticos, y menos las causas y mecanismos de las enfermedades. La competencia entre compañías farmacéuticas por alcanzar la patente de un nuevo medicamento ha sido un nuevo fin de la investigación. Asimismo, se ha favorecido investigar pequeñas diferencias entre medicamentos, buscando la aprobación de moléculas semejantes, por la ventaja económica que puede redituar al obtener la patente de la nueva $(3,6)$.

Además, los ensayos clínicos y las guías basadas en evidencias han dado origen, influenciados por las ganancias, a un cambio en las estadísticas de salud-enfermedad. Así, por ejemplo, al ser muy rentables los medicamentos antihipertensivos o los reductores de colesterol, los ensayos han demostrado nuevos límites de presión arterial o colesterol, en orden a fomentar un exceso de prevención y consumo de medicamentos. Así ha surgido la pre hipertensión por una expansión en los rangos para diagnosticar hipertensión, con la recomendación de llevar dieta o ejercicio, o medicarse; mucha gente, sabiéndose enferma, opta por lo segundo. De igual manera, las cifras de hipercolesterolemia han ido bajando desde $280 \mathrm{mg} / 100 \mathrm{ml}$ hasta $200 \mathrm{mg} / 100 \mathrm{ml}$ (o menos). Como a mucha gente le es difícil llevar una dieta rigurosa y un plan de ejercicio, optará por tomar medicamento $(2,10)$.

En definitiva, la vinculación entre el interés económico de la IF y su patrocinio de los ensayos clínicos trajo como consecuencia que los investigadores que forman parte de los ensayos clínicos de la IF no sean independientes, salvo que existan reglas muy claras al respecto. Su vinculación con la IF no solo ha incluido subsidios sino también la participación en otros arreglos financieros.

"Los investigadores se desempeñan como consultores de las compañías cuyos productos están estudiando, integran los comités asesores y las mesas de conferencias, hacen arreglos de patentes y derechos, aceptan figurar como autores de artículos ajenos que reflejan los intereses de las compañías, promueven fármacos y dispositivos en congresos auspiciados por la compañías y se permiten recibir costosos regalos y viajes a sitios lujosos"(10:1516). 
Los ensayos clínicos internacionales al servicio de la estrategia comercial global de la IF

Los ensayos clínicos multicéntricos internacionales o proyectos de investigación multinacionales multicéntricos (PIMM) son una modalidad reciente de investigación internacional, cuyo diseño es realizado en países con gran capacidad tecnológica y de investigación, particularmente por corporativos transnacionales que cuentan entre sus negocios con grandes firmas farmacéuticas, y cuya aplicación es llevada a cabo en diversos países y centros hospitalarios del mundo.

Los PIMM muestran importantes conflictos de interés financiero que enturbian su supuesta neutralidad científica. Esto debido a que el financiamiento de los ensayos clínicos proveniente de la IF influye en la conducción y resultados de los mismos, en tanto que su financiamiento y conducción se ha dejado cada vez más en manos de grandes corporativos transnacionales(8).

Siguiendo los resultados de $\operatorname{Bodenheimer}(11)^{7}$, se pueden confirmar algunas de las afirmaciones hechas respecto a la IF:

- Con el nuevo énfasis en la prevención y tratamiento de enfermedades crónicas, ha cambiado la investigación en medicamentos. Para establecer la eficacia y la seguridad de este tipo de medicamentos, los ensayos clínicos necesitan ser largos, duraderos y realizados en múltiples centros, puesto que un solo centro no puede reclutar el número suficiente de pacientes para asegurar la validez estadística ${ }^{8}$.

- Para acelerar los ensayos clínicos, la industria está cambiando de los centros médicos académi-

\footnotetext{
${ }^{7}$ Bodenheimer realizó un estudio para discutir los problemas relacionados con el financiamiento de los ensayos clínicos multicéntricos por redes comerciales. Entrevistó a 39 participantes de diversas maneras en los ensayos clínicos: 6 ejecutivos farmacéuticos, 12 investigadores clínicos, 9 de oficinas de investigación de universidades, 2 médicos de las redes comerciales de medicamentos, 8 personas que han estudiado el proceso de los ensayos clínicos de medicamentos y 2 escritores médicos profesionales. La entrevista consistió en preguntas estándar y una oportunidad de los entrevistados de discutir sobre la relación industria-investigador de manera general.

${ }^{8}$ Habría que añadir que, al investigar la IF en países pobres, se beneficia por la gran población que le promete un amplio reclutamiento de pacientes. Además, en estos países hay que pasar menos trámites regulatorios burocráticos para licitar los PIMM, en comparación con los países ricos.
}

cos a organizaciones lucrativas de mercado (CROs y site-management organizations, $\operatorname{SMOs})(8,12)$, que contratan a médicos, científicos, farmaceutas, especialistas en estadística y gerentes, y ofrecen a la IF un menú de servicios. La competencia por el dinero producto de los ensayos se ha configurado como cientos de CROs y SMOs, centros médicos académicos y sitios no académicos independientes "luchando por la parte más grande del pastel". Se les critica que se sobrecomprometen, pero que cumplen menos de lo que pueden. También se les objeta su preocupación por la aprobación y lanzamiento al mercado de los medicamentos, más que por la creación de ciencia.

- Respecto del diseño de los protocolos, hace ańos los investigadores ajenos a los ensayos diseñaban los protocolos de investigación, pero actualmente es la IF quien hace los ensayos y contrata a investigadores para dar su firma, sin posibilidades de modificación?

- En relación con la autoría, antes escribía un autor principal, pero actualmente se está dando el síndrome autor no escritor/no autor escritor (non writing autor/non author writer). El síndrome tiene dos facetas: un escritor médico profesional (escritor fantasma), contratado por una CRO o la industria y pagado por escribir el artículo a partir de unos materiales que recibe, pero no es llamado como autor; y un investigador clínico (escritor invitado) que aparece como autor pero no analiza los datos ni lee el manuscrito, aunque tiene acceso a él $(7,11)$.

Pero además los conflictos de interés financiero son producto de la lógica del mercado mundial. La IF mundial está dominada por un pequeño número de firmas multinacionales, su investigación obedece más a las leyes del mercado que a las prioridades médicas o necesidades sociales del lugar donde se investiga, y hace inversiones para maximizar el valor actual de futuras ganancias a partir del dinero invertido. La investigación far-

\footnotetext{
${ }^{9}$ Se mencionan posibles variaciones en los ensayos que pueden introducirse para favorecer la aprobación del medicamento: probar el nuevo medicamento en población más joven, logrando mejores resultados y menos efectos adversos que en personas mayores; comparar el nuevo medicamento con una dosis menor del producto en competencia, originando que el nuevo parezca más eficaz; utilizar end points subrogados que pueden no coincidir con los clínicos, para favorecer al medicamento prueba.
} 
macéutica está sobre todo abocada al desarrollo de nuevos medicamentos y se lleva a cabo principalmente en el sector privado.

Según W. Comanor(13), en un anexo del informe Research \& Development de la Organización Mundial de la Salud (OMS), de 1996, la concentración geográfica de la investigación farmacéutica en pocos países ricos afecta a la innovación y la investigación mundial. Los países ricos albergan a las compañías farmacéuticas con mayor poder de realizar investigación a fondo y actividades de desarrollo. En dichos países, el consumo mundial agregado de medicamentos es del $80 \%$ respecto del consumo de los países pobres, que es del $20 \%$. Desde una base per cápita, la diferencia es aún mayor, con un cociente de 18 a 1 . La diferencia en el consumo no estriba en las preferencias, sino en la capacidad de consumo de los países.

El costo de la producción y aprobación de un nuevo medicamento es aproximadamente de 25 millones de dólares, lo cual lleva a la IF a colocar sus nuevos productos según la demanda prospectiva de estos. Sin embargo, de los 1.393 medicamentos aprobados en el mundo entre 1975 y 1999 , solo 13 se destinaron a la cura de enfermedades tropicales(14). Por lo tanto, la investigación de las multinacionales está orientada por el mercado y no por necesidades médicas o conocimientos científicos, mientras que las necesidades de la población de los países pobres son sacrificadas.

Por otra parte, como se ha dicho, cada vez más la IF favorece el consumo de medicamentos en orden a obtener ganancias y genera proyectos de investigación determinados por el interés económico, aun en los países centrales como EU, debido a su capacidad de consumo(15). En los países ricos, la IF ha dado origen a la investigación en las llamadas "me too drugs", es decir, medicamentos estructuralmente muy parecidos a otros ya aprobados, compitiendo por conquistar nichos atractivos para el mercado. Del mismo modo, se ha incrementado la investigación en medicamentos para incrementar la calidad de vida o aliviar los síntomas del envejecimiento, las así llamadas "lifestyle drugs" (16).

Los laboratorios hacen grandes inversiones pero buscan un rápido retorno de su dinero. Un moti- vo atrayente para la realización de ensayos clínicos por la IF es el factor tiempo. Cada día de retraso en la aprobación de una patente le cuesta a la industria aproximadamente USD $\$ 1.300 .000$, por lo tanto entre más rápido pueda llevarse a cabo la aprobación del medicamento, será más beneficioso. Esto resulta particularmente factible con las fases II y III tardías de los ensayos clínicos, las cuales pueden acelerarse reclutando un gran número de pacientes de varios países y centros a la vez, obteniendo como resultado un periodo breve de tiempo para reunir la información necesaria y obtener así la patente deseada lo antes $\operatorname{posible}(17)^{10}$.

Hay que mencionar también algunas constataciones sobre las investigaciones de la IF en otros países(8). La IF se beneficia al realizar investigaciones en países periféricos o emergentes, puesto que le cuesta menos realizar los ensayos clínicos: entre 1995 y 2005 , el número de países huéspedes de ensayos multinacionales ha crecido al doble, mientras que la proporción de ensayos en EU y Europa ha disminuido. Los ensayos clínicos representan dos tercios del costo invertido en $\mathrm{I}+\mathrm{D}$ de un medicamento, por lo que economizar en esta área representa un importante ahorro. A la investigación farmacéutica globalizada se le ha llamado el "nuevo oro" y se le ha considerado como una subasta en la que varios países compiten por lograr la realización de PIMM en su territorio ante diversas ofertas de la IF $\operatorname{global}(8,9)$.

Sin embargo, ni para los pacientes ni para el ejercicio de la medicina en las comunidades huéspedes se observa una ventaja proporcional a la obtenida por la IF por realizar sus investigaciones en países periféricos, tal como lo muestran los precios de los nuevos medicamentos. México, país emergente, con una gran cantidad de pobres, ocupa el primer lugar en América Latina como mercado farmacéutico y es el lugar más caro de la región en el costo de tratamientos $(8,18)$. Los datos empíricos de cientos de pacientes que no pueden surtir su receta médica por los inalcanzables precios de los nuevos medicamentos es un

\footnotetext{
${ }^{10}$ Una razón técnica de los ensayos clínicos internacionales es acumular casos para calcular densidades de incidencia, lo cual se puede prestar a lo siguiente: en lugar de estudiar 20 casos en dos años ( 40 años por persona), mejor estudiar 40 casos en un año (también 40 ańos por persona), lo cual puede afectar la observación de efectos crónicos.
} 
dato cada vez más del diario acontecer en la práctica médica nacional. Realizar PIMM para descubrir nuevos productos en países en los que, a excepción de las elites, la mayoría de la población no podrá adquirirlos, o en centros de medicina pública que se debaten entre el recorte de presupuesto y la excesiva demanda de atención médica, parece una incoherencia.

\section{A la búsqueda de nuevas fronteras}

De manera creciente, los ensayos clínicos controlados tienen un apartado destinado a la farmacogenética, buscando además del objetivo planteado en el protocolo, investigar aspectos farmacogenéticos de determinada molécula. No se trata ya de reemplazar los genes defectuosos, como es el caso de la terapia génica, sino de suministrar nuevas proteínas blanco que sirvan para "screenear” moléculas clásicas (pequeñas moléculas). Se espera poder intervenir en las primeras formas de expresión del gen en las células y, por tanto, mucho antes de la acción de los medicamentos actualmente disponibles. Estos blancos biológicos receptores serán productos de expresión del gen defectuoso, susceptibles ahora de ser bloqueados selectivamente. Vendrían a engrosar el stock disponible de los 400 blancos utilizados hoy para el screening por la $\operatorname{IF}(3)$.

En el futuro, la farmacogenética influirá indudablemente la manera en que las nuevas moléculas transitarán las fases clínicas de los ensayos por la IF y, eventualmente, serán registradas como medicamentos en el mercado. Aunque los desarrollos de la farmacogenética pueden originar medicamentos blockbuster, también pueden rescatar y vender medicamentos antes terminados en fases tardías del desarrollo clínico por su toxicidad, o crear medicamentos mucho más efectivos que sus competidores. También, debido al sistema de exclusividad de patentes de Estados Unidos, el hallazgo de un marcador farmacogenético puede hacer ganar a una firma extensiones de la patente o periodos adicionales de exclusividad del mercado. Y mientras que una estructura molecular de un medicamento patentado se hace pública y se envía a las autoridades regulatorias, no es así en el caso de un test farmacogenético propiedad de la IF. Esto último significa que los producto- res de medicamentos genéricos pueden generar la nueva molécula, pero no podrán venderla sino en relación con el test farmacogenético, a menos que desarrollen sus propios ensayos clínicos para una versión genérica del test farmacogenético.

Por último, la farmacogenética también es un negocio internacional: los PIMM cada vez más traen una sección específica para ello. Aun antes de que las compañías de biotecnología puedan buscar mercados entre los poderosos, ellas necesitan poblaciones en las que investigar, que hoy abundan en los países pobres. La investigación internacional a menudo apunta a poblaciones relativamente aisladas, cuya reserva genética provee una útil manera de estudiar la enfermedad, y cuya falta de poder les hace menos capaces de negociar una compensación o solicitar una más justa distribución de beneficios o ganancias. Más aún, la investigación en otras tierras permite a los patrocinadores evadir normas de protección de los sujetos que en sus países son más estrictas $(19,20)$.

Se preven dos escenarios a futuro nada halagadores, puesto que apuntan a la exclusión de las mayorías: a) negocios, como siempre, en los que el principal objetivo sería desarrollar conocimiento útil en el nivel individual, para predecir toxicidad o efectividad de los medicamentos, las poblaciones en los ensayos no son seleccionadas por pruebas farmacogenéticas y las nuevas moléculas son eventualmente registradas para uso general. Los privilegios de los países ricos pueden "caer" sobre los países pobres, con un considerable retraso conforme se vaya extendiendo su uso, reforzando los esquemas de dependencia comercial; b) cambio fundamental: se trata de una reconfiguración de los procedimientos de desarrollo de los medicamentos y los tests farmacogenéticos que serían usados para restringir el grupo de pacientes enrolados en los ensayos clínicos fase III y IV, tanto a aquellos para quienes es probable que la molécula será efectiva o para quienes es probable que no existirá toxicidad. El medicamento es probado en subpoblaciones genéticamente definidas, y las nuevas moléculas son registradas solo para uso en personas con un genotipo específico. En este caso, el conocimiento generado será solo para poblaciones selectas (21). 


\section{Conclusión}

La investigación de la IF está muy condicionada por el interés económico, lo que hace difícil mantener la integridad y sobre todo la pertinencia de la investigación científica, pues esta obedece no sólo a razones científicas, sino de mercado. La relevancia de la pregunta de investigación se relativiza frente al interés mercantil, tomando poco o nada en cuenta las necesidades sociales del lugar donde se investiga. Además, la supuesta neutralidad científica ha quedado en ocasiones en entredicho al constatar sesgos en las publicaciones de resultados. Mientras no se mantenga una independencia en la investigación, su ser y su finalidad estarán mancilladas por el interés económico.

Los ensayos clínicos internacionales son una expresión de lo anteriormente dicho, puesto que su financiamiento y su conducción están influenciados desde redes comerciales que, de una manera u otra, afectan los resultados y los fines de la investigación. Los PIMM son parte de una estrategia mundial cuya máxima es optimizar las ganancias compitiendo por innovar medicamentos. Para ello es necesario contar con numerosos pacientes, distribuidos en varios centros a lo largo del mundo, enrolados en protocolos cuya finalidad muchas veces es lanzar nuevas moléculas cada vez mejor rentables en el mercado mundial en el menor tiempo posible.

Este escenario contradictorio, en el cual el dominio del mercado empaña los fines de la investigación, se repite y tiende a exacerbarse en el caso de la farmacogenética, la cual se ha desarrollado como parte de los PIMM para encontrar nuevas terapias, pero que serán solo para quienes puedan pagarlas o para elites selectas.

El interés económico condiciona de diversas maneras la integridad científica de la investigación, lo cual requiere ser considerado desde diversos frentes en vistas a devolverle a la investigación científica su neutralidad y su orientación al bien común.

\section{Referencias}

1. Drews J. In Quest of Tomorrow's Medicines. New York: Springer; 2003.

2. Angell M. The Truth About the Drug Companies. How They Deceive Us and What To Do About It. New York: Random House; 2004.

3. Pignarre P. El gran secreto de la industria farmacéutica. Barcelona: Gedisa; 2005.

4. Freeman RA. Industry perspectives on equity, access, and corporate social responsibility: a view from the inside. In: Cohen JC, Illingworth P, Schüklenk U, (eds). The Power of Pills. Social, Ethical \& Legal Issues in Drug Development, Marketing \& Pricing. London: Pluto Press; 2006: 65-73.

5. Appelbaum K. Is Marketing the Enemy of Pharmaceutical Innovation? Hastings Center Report 2009; 39(4): 13-17.

6. Bekelman J, Mphil Y, Gross C. Scope and Impact of Financial Conflicts of Interest in Biomedical Research. The Journal of the American Medical Association 2003; 289(4): 454-465.

7. Fugh-Berman A. The Haunting of Medical Journals: How Ghostwriting Sold "HRT". PLoS Medicine 2010; 7(9) $1-11$.

8. Petryna A. When Experiments Travel. Clinical Trials and the Global Search for Human Subjects. New Jersey: Princeton University Press; 2009.

9. London A. Research at the Auction Block: Problems for the Fair Benefits Approach to International Research. Hastings Center Report 2010; 40(4): 35-45.

10. Angell M. Is Academic Medicine for Sale? The New England Journal of Medicine 2000; 342(20): 1516-1518. 11. Bodenheimer T. Uneasy alliance - Clinical Investigators and the Pharmaceutical Industry. The New England Journal of Medicine 2000; 342(20): 1539-1544.

12. Conseil National du Sida. Avis sur les problèmes éthiques posés par la recherche clinique dans les pays en développement. Paris; Mars 2003. Consultado: 2010 oct 17. Disponible en: http://www.cns.sante.fr/spip. php?article201\&artpage $=2-7$

13. Comanor WS. World Health Organization. Annex 4: The Pharmaceutical Industry and the Health Needs of Developing Countries. In: Ad Hoc Committee on Health Research Relating to Future Intervention Options, Investing in Health Research and Development. Geneva: 1996. (Document TDR/Gen/96.1). 
14. Pogge T. A new approach to pharmaceutical innovation. Australia; June 2005. Consultado: 2010 oct 16. Disponible en: http://www.onlineopinion.com.au/view.asp?article=3559

15. Lemens T. Piercing the Veil of Corporate Secrecy about Clinical Trials. Hastings Center Report 2004; 34(5): $14-18$.

16. Luna F. Algunas controversias acerca de las guías internacionales para la investigación con seres humanos. Jurisprudencia Argentina 2004; IV: 36-42.

17. Glickman SW, Hutchinson JG, Peterson ED, Cairns ChB, Harrington RA, Califf RM, et al. Ethical and Scientific Implications of the Globalization of Clinical Research. The New England Journal of Medicine 2009; 360(8): 816-823.

18. Organisation for Economic Cooperation and Development (OCDE). Pharmaceutical Pricing and Reimbursment Policies in Mexico. Paris; 2007. Consultado: 2010 oct 16. Disponible en: http://www.oecd.org/dataoecd/39/36/38097348.pdf

19. Sowle L. Genetics, Commodification and Social Justice in the Globalization Era. Kennedy Institute of Ethics Jorunal 2001; 11(3): 221-238.

20. Mwase I. Genetic Enhancement and the Fate of the Worse Off. Kennedy Institute of Ethics Journal 2005; 15(1): 83-88.

21. Holm S. Pharmacogenetics and global (in)justice. In: Cohen JC, Hillingworth P, Schüklenk U, (eds). The Power of Pills. Social, Ethical \& Legal Issues in Drug Development, Marketing \& Pricing. London: Pluto Press; 2006: 98-105.

Recibido: 12 de enero de 2011

Aceptado: 17 de marzo de 2011 